\title{
Equality, Diversity and Inclusion report published
}

The 'Equality, Diversity and Inclusion within Dentistry - a profession wide commitment' report has been published by the Diversity in Dentistry Action Group (DDAG).

It marks the DDAG's commitment to collaborative action and shows the dental organisations which have pledged to some key principles for change, including actively committing to addressing racism and discrimination, exploring and developing opportunities for representation and inclusion, and organisational commitment to change at all levels.

Chair of the Group, GDP and Clinical Leadership Manager for the Office of the Chief Dental Officer, Nishma Sharma says: 'The issue of racism, prejudice, bias is an outdated, stagnant corroded bolus, stuck and wedged into the throat of society; choking progression, suffocating innovation, stifling the potential of a fresh representative workforce where there are no glass ceilings.

'This can no longer be ignored. Its deep roots are steeped in history, entrenched in the many conflicts and injustices endured over the years. It is now time to break down the barriers using the armoury of education, understanding, empathy, proactive reaction, energy, insight and allyship. Together we are a profession, yes, but we are also individuals with a responsibility for change within our own spheres of influence and extending to wider society'

Chief Dental Officer for England, Sara Hurley, added: 'I have asked the action group to garner the seldom heard, to identify and acknowledge the conscious and unconscious bias, to challenge the system to listen and act, to work collaboratively across the profession to co-ordinate sustained action.

'This publication, and the commitments from each and every one of the organisations that have contributed to our call to action, sets the scene for sustainable change; from practice level to corporate boards, from dental policy to dental public health, from academic arenas to regulation. These organisations and their members, by virtue of their position and influence on the profession's culture, values and ethics, have publicly placed themselves in the vanguard of change, fostering greater equality, diversity and inclusion.'

Commenting on the report, Laura Cross and Shareena Ilyas, Co-Chairs of the BDA's Equality, Diversity and Inclusion Committee said: 'We are proud to have contributed to this new report from the DDAG and applaud their collaborative approach to tackling racism in the dental profession. Only together can we break down the barriers that are at the root of discrimination and ensure that everyone has the opportunity to pursue a successful career in dentistry.

'We look forward to continuing to help drive this agenda forward and to make dentistry a more inclusive and equitable profession. We are continuing with our work on equality, diversity and inclusion, investigating the issues faced within dentistry, and helping to define the solutions.

\section{Dental Complaints Service performance 'highest level on record'}

The Dental Complaints Service (DCS) has revealed $98 \%$ of enquiries were responded to within two days - a four per cent improvement on the previous year and highest level on record. In its 2020 performance report, the DCS cite their high performance came in a challenging year due to COVID-19 complications and when total complaint numbers $(3,159)$ topped those received in each of the previous five years.

Other notable findings in the report include:

$\rightarrow$ The most common issues raised related to perceived failure of treatment (85\%). Within that, the type of treatment that attracted most complaints related to removable orthodontic appliances, reflecting a rise in the number of patients seeking remote orthodontics

$\rightarrow$ A number of complaints relating to COVID-19 were received. These included not being informed in advance of charges for Personal Protection Equipment (PPE), a lack of NHS appointment availability or no NHS PPE and, as a result, being encouraged to go private or wait longer for an NHS appointment $\rightarrow$ The number of fitness to practise referrals from the DCS fell to 36 in 2020, 24 of which related to just three dental professionals

$\rightarrow 600$ complaints were received in relation to the collapse of one dental corporate, which closed its doors leaving many patients who had paid in advance out of pocket. The DCS continues to work with stakeholders around the challenges presented by some corporate structures.

Head of DCS Operations, Michelle Williams said: 'For many people, 2020 represents the most challenging year in living memory and I would like to thank all those we have worked with - the dental professionals, the stakeholder organisations and most of all the DCS team - for their hard work and resilience in the face of such adversity. This report provides useful information for everyone involved in the provision of dental services, but particularly so for dental professionals as there are useful insights for consideration in everyday practice.'

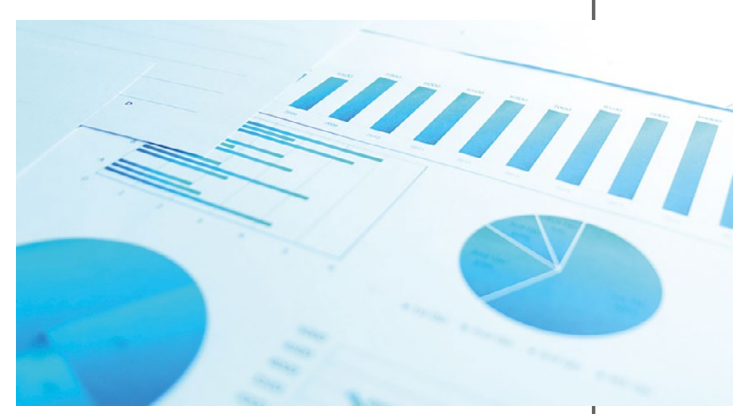

Dr Len D'Cruz, Head of BDA Indemnity, said: 'The 2020 DCS Review contains a lot of information but, if one point stands out above all else, it is that when a patient complains about private treatment, the dentist involved would be strongly advised to attempt local resolution.

'BDA Indemnity considers the DCS conciliation process to be very fair and welcomes their practical approach to working with indemnity providers to contain the involvement of the dentist, leaving an experienced dento-legal team to handle the matter whilst the clinician can continue to work.' 